\title{
HISTOCHEMICAL LOCALIZATION OF ALKALINE PHOSPHATASE IN THE RETINA*
}

\author{
BY \\ J. L. REIS \\ Postgraduate Medical School, London
}

CHEMICAL investigations have shown that alkaline phosphatase is present in the retina (De Conciliis, 1934; Reis, 1951). In these investigations the whole retina was homogenized and the enzyme estimated in extract. Obviously the estimations of enzyme activity by such a necessarily gross method give no idea of the exact distribution of the enzyme in this, functionally, so finely differentiated tissue. This object may only be achieved by using histochemical methods. A knowledge of the histological localization of the phosphatase in the retina, may perhaps enable us to make some suggestions about its physio logical role there.

Gomori (1939) described a method of demonstrating alkaline phosphatase activity in tissue slides. This method consists in incubating the fixed tissue slides with buffered solution of a phosphate ester and a calcium salt. The phosphatase hydrolyses the ester, and the liberated phosphate forms a precipitate of calcium phosphate. The latter is converted to cobalt phosphate (by the addition of cobalt nitrate solution) and this with ammonium sulphide produces black, insoluble, cobalt sulphide. Thus black deposits of cobalt sulphide are formed at the sites of phosphatase activity.

This technique was used by Süllmann and Payot (1949) in the study of corneal epithelium phosphatase. François and Rabaey (1951), and simultaneously De Vincentiis (1951), published their investigations on the phosphatase activity of different ocular tissues. Since in the described method phosphatase activity is visualized as a brown or black deposit, it is difficult to differentiate it from the similarly coloured melanin pigment. Francois and Rabaey comment on this difficulty in their paper. They make the final observations by comparing the phosphatase-stained slides with control slides. If melanin is present in the control, the activity of phosphatase is demonstrated when a darker or more abundant staining seems apparent in the incubated slide. De Vincentiis (1951) found another solution to this problem. For the study of phosphatase in uveal membranes he used albino rabbits-thus avoiding the presence of melonin, but limiting his research to these animals only.

The disadvantage of the method used in these papers is that calcium phosphate is not wholly insoluble but is apt to be diffused to a certain extent. As a result, deposits may occur which are not a true indication of phosphatase activity. There also exists a tissue affinity for cobalt salts, so that the cobalt

- Received for publication June 16, 1953. 
salts may not only react with the calcium phosphate but may also be attached directly to some tissue structures. In order to avoid these difficulties, Gomori (1951) devised a new method of phosphatase staining, subsequently modified by Grogg and Pearse (1952). This new method consists in using $\alpha$-naphthylphosphate in the presence of a diazonium compound. The $\alpha$-naphthol produced by the phosphatase hydrolysis forms a diazo-dye with the diazonium compound. If the diazotate of 5-chloro-o-toluidine is used, the resulting diazo-dye is red and easily differentiated from the melanin pigment. It does not diffuse so easily as calcium phosphate. If this method is used, the nuclei usually do not stain. This suggests that what was previously often described as nuclear phosphatase, was an artefact, due most probably to the diffusion of calcium phosphate.

In all previous papers dealing with histochemistry of eye-tissue phosphatases, the Gomori's first method was used and a great deal of nuclear staining was observed. It therefore seemed that it could be of interest to apply this new method to eye tissues, in this way excluding the diffusion of calcium phosphate and the unspecific cobalt staining, and giving the resulting deposits a different colour from that of the melanin pigment.

\section{Method}

Gomori's method was used for tissue fixation (cold acetone) and for the preparation of slides as described by Pearse and Reis (1952). The slides were incubated by the method of Grogg and Pearse (1952) and Pearse (1953) at room temperature for 15 to $30 \mathrm{~min}$. in freshly-made solution containing $1 \mathrm{mg}$. sodium $\alpha$-naphthylphosphate and $1 \mathrm{mg}$. 5-chloro-o-toluidine diazotate per $1 \mathrm{ml} . \mathrm{m} / 20$ veronal buffer $(p \mathrm{H} 9)$.

Some slides were stained using for comparison Gomori's phosphatase method (calcium phosphate-cobalt sulphide).

\section{Results}

For comparison a slide of bovine retina was stained by Gomori's phosphatase method (Fig. 1).

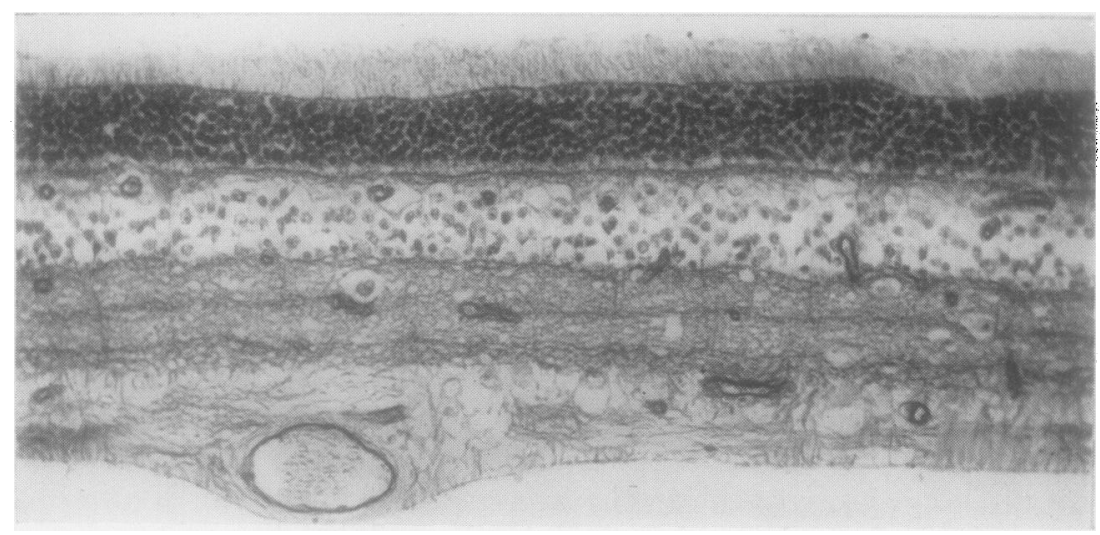

FIG. 1.-Alkaline phosphatase in bovine retina using the calcium phosphatecobalt sulphide method. $\times 190$. 
Fig. 2 shows a serial slide of the same retina stained by the new diazo-dye phosphatase method. The difference is clear, whereas the old method shows extensive staining of the nuclei in addition to staining of the vascular endothelia and of the plexiform layers; in the diazo-dye method only the vascular endothelia are well stained, the plexiform layers show a very slight reaction, and the nuclei do not stain at all.

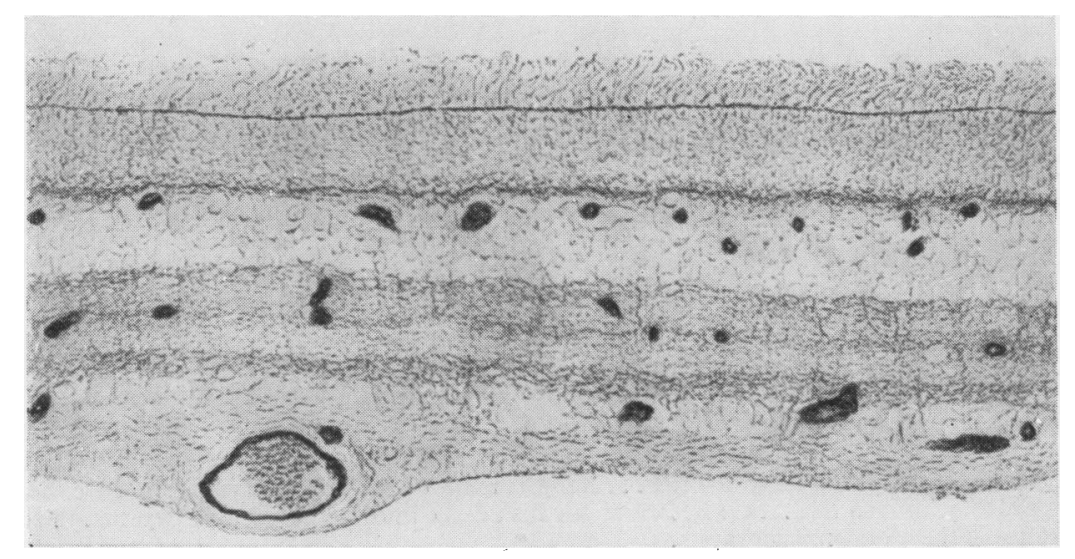

Fig. 2.-Alkaline phosphatase in bovine retina using the diazo-dye method. $\times 190$.

\section{Conclusions}

François and Rabaey (1951) and De Vincentiis (1951), studying the histological localization of alkaline phosphatase, show very marked staining of nuclei. As they were using Gomori's method, which he himself (Gomori, 1951) later decided was not very accurate, it is probable that their results were affected by the calcium phosphate diffusion. The nuclei in general have affinity for different substances, e.g. dyes such as methylene blue. The affinity of nuclei for the calcium phosphate is probably of a similar kind. The final cobalt sulphide deposits do not correspond here exactly to the sites of phosphatase activity. The diazo-dye method seems to be much more selective and freer from diffusion phenomena, and its results seem more reliable than those obtained by the older methods.

The staining of the vascular endothelium in the retina, using the alkaline phosphatase diazo-dye method, is so sharply defined that it seems to be possible to apply it to the study of the anatomy and pathology of the retinal vessels.

Some earlier writers, basing their observations of the phosphatase activity in the retina on chemical estimations, suggested that the alkaline phosphatase in the retina may play a role in phosphate metabolism connected with photochemical reactions. In view of the histological localization of phosphatase, this does not seem possible, all the enzyme being concentrated in the inner walls of the vessels. 
It seems worth mentioning here that a highly active alkaline phosphatase is present in the whole uveal membrane and in the choroid plexus. These tissues play some part in the formation of the intra-ocular and cerebrospinal fluids, in that they regulate to a certain degree the passage of ions. It follows that the alkaline phosphatase prơtbably has a role in regulating the phosphate ion concentrations. The site of its activity in the eye corresponds to the hypothetical "blood-aqueous barrier".

\section{Summary}

(1) Alkaline phosphatase localization in the retina was studied by the diazo-dye coupling method.

(2) Alkaline phosphatase in the retina was found mainly in the vascular endothelium, only a very slight reaction being shown by the plexiform layers.

(3) The extensive staining of retinal nuclei when Gomori's phosphatase method (calcium phosphate-cadmium sulphide) is used, is an artefact due to calcium phosphate diffusion.

I wish to express my thanks to Prof. E. J. King for the opportunity to perform the experiments n his laboratory, and to Dr. A. G. E. Pearse for his advice and the necessary reagents. Mr. E. W. Willmott took the photomicrographs.

DE ConcILIIS, N. (1934). Sperimentale, 88, 793.

De Vincentus, M. (1951). Arch. Ottal., 55, 303.

Françors, J., and RABAeY, M. (1951). Ann. Oculist. (Paris), 184, 481.

GoMORI, G. (1939). Proc. Soc. exp. Biol. (N.Y.), 42, 23.

GroGg (1951). J. Lab. clin. Med., 37, 526.

Grogg, E., and Prarse, A. G. E. (1952). Nature (Lond.), 170, 578.

PEARSE, A. G. E. (1953). "Histochemistry". Churchill, London. and ReIS, J. L. (1952). Biochem. J., 50, 534.

REIS, J. L. (1951). British Journal of Ophthalmology, 35, 149.

SüllmanN, H., and PAYOT, P. (1949). Ophthalmologica (Basel), 118, 345. 